\title{
The Role of Nickel in Radiation Damage of Ferritic Alloys.
}

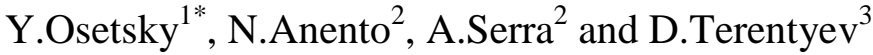

${ }^{1}$ Materials Science and Technology Division, Oak Ridge National Laboratory, Oak

Ridge, TN 37831, USA.

${ }^{2}$ Departament Matemàtica Aplicada III, E.T.S. Enginyeria de Camins, Universitat

Politècnica de Catalunya (UPC). BarcelonaTech, Jordi Girona 1-3, 08034 Barcelona, Spain

${ }^{3}$ SCK.CEN, Nuclear Materials Science Institute, Boeretang 200, B-2400 Mol, Belgium

\begin{abstract}
According to the modern theory damage evolution under neutron irradiation depends on the fraction of self-interstitial atoms (SIAs) produced in the form of one-dimensionally glissile clusters. These clusters, having a low interaction cross-section with other defects, are absorbed mainly by grain boundaries and dislocations creating the so-called production bias. It is known empirically that addition of certain alloying elements influence many radiation effects, including swelling, however the mechanisms are unknown in many cases. In this paper we report the results of an extensive multi-technique atomistic level modeling study of SIA clusters mobility in bcc Fe-Ni alloys. We have found that $\mathrm{Ni}$ interacts strongly with the periphery of clusters affecting their mobility. The total effect is defined by the number of $\mathrm{Ni}$ atoms interacting with the cluster at the same time and can be significant even in low-Ni alloys. Thus $1 \mathrm{~nm}$ (37SIAs) cluster is practically immobile at $\mathrm{T}<500 \mathrm{~K}$ in the Fe-0.8at.\% Ni alloy. Increasing cluster size and Ni content enhance cluster immobilization. This effect should have quite broad consequences in void swelling, matrix damage accumulation and radiation induced hardening and the results obtained help to better understand and predict the effects of radiation in Fe-Ni ferritic alloys.
\end{abstract}

Keywords: Dislocation loops, Fe-Ni alloys, Diffusion mechanism, Radiation effects. 


\section{Introduction}

Growing energy needs demand significant improvement in efficiency of energy production including the usage of nuclear power plants [1]. Prediction of the behavior of structural materials under operational irradiation conditions is strongly wanted for it is crucial for safe long term operation [2]. However, extending nuclear plants lifetime makes direct experimental predictions impossible for the experiments must last decades to correctly reproduce the corresponding radiation effects. Therefore, theoretical and computational approaches with predictive capabilities become commercially important applications. Any practically useful prediction that exceeds available experimental data should be a) based on the best available theory and b) realized as a computational approach through the models of necessary scales. The modern approach to generalized radiation damage theory, described in [3], is able to explain qualitatively and, in many cases, quantitatively radiation-induced phenomena such as the effects of recoil energy spectra and grain boundaries on swelling; void ordering and void lattice formation; radiation induced segregation and radiation growth in anisotropic materials [3-10]. At the moment this theory is the most promising candidate to provide the basis for predictive modeling of materials behavior under irradiation. According to [3], in the commercially important case of cascade-type damage (neutron, ions) and for a material with a low dislocation density, $\rho_{d}$, the steady state rate of swelling $S$ is independent on the irradiation dose $\Phi$ and can be expressed as:

$$
\frac{d S}{d \emptyset} \approx\left(1-\varepsilon_{r}\right) \varepsilon_{i}^{g} k_{c}^{2} /\left(k_{c}^{2}+Z_{V}^{d} \rho_{d}\right) \approx\left(1-\varepsilon_{r}\right) \varepsilon_{i}^{g}
$$

where $\left(1-\varepsilon_{\mathrm{r}}\right)$ is the fraction of NRT-estimated defects (for NRT standard see [11]) surviving after cascades, $\varepsilon_{i}^{g}$ is the fraction of self-interstitial atoms (SIAs) created in the form of onedimensionally (1-D) glissile clusters, $k_{c}^{2}$ is the total sink strength in the system and $Z_{V}^{d} \approx 1$ is the 
dislocation efficiency for absorbing vacancies. The swelling rate is proportional to $\varepsilon_{i}^{g}$ because 1D glissile clusters have a very low interaction cross-section with other defects. As a result they do not recombine with vacancies in the bulk but have a long mean free path (of the order of $\mu \mathrm{m}$ ) and usually are absorbed by grain boundaries or dislocations which increases the vacancy supersaturation. This is called "production bias" and it appears exclusively due to a) direct formation of SIA clusters under cascade damage conditions and b) ability of interstitial clusters to glide one-dimensionally. Production bias together with the conventional "dislocation bias" defines radiation swelling and many other radiation effects. Eq.(1) looks quite simple but describes the most important consequence of cascade-type irradiation and has successfully explained the recoil energy effect on swelling [4] from the contribution of the production bias. Eq.(1) presents the limit case when SIA clusters glide fast i.e. the known case of perfect pure metals. In the more general i.e. when cluster's glide speed can be affected by the local environment, the production bias contribution depends on the cluster mean free path that, in its turn, is defined by the cluster diffusivity. It is therefore obvious that by affecting the formation of glissile clusters and/or their 1-D motion one can control a significant contribution to the radiation swelling and other related effects. Note that addition of certain alloying elements has been successfully used over years to control many radiation effects, including swelling, in a purely empirical way.

In this paper we report our results from a study of the effect of nickel impurity in iron on 1-D glide of interstitial clusters/dislocation loops. There are several reasons for the choice of this system. First, ferritic alloys are widely used structural material in nuclear energy. Second, Ni is known for its ability to stabilize gamma-Fe phase in stainless steels. However, the knowledge of its effect on radiation resistance in ferritic steels is rather limited: There is some evidence that $\mathrm{Ni}$ 
affects $\mathrm{Cu}$-precipitate evolution [12], dislocation loop populations [13], mechanical properties [14] and, possibly, swelling [15]. Third, the set of interatomic potentials available Fe-Fe [16], Fe-Ni [17] and Ni-Ni [18] provides a very realistic description of $\mathrm{Ni}$ in ferritic alloys due to a thorough fitting to a number of ab initio properties in the Fe-Ni system. Moreover, a recent multi-technique $a b$ initio study of Ni-impurity interactions with screw and edge dislocations in Fe [19] has confirmed the high accuracy of the Fe-Ni potential from [17]. This is because Ni in $\mathrm{Fe}$ is in a paramagnetic state and the magnetic effects, that complicate the description of $\mathrm{Cr}$ or Mn impurity in $\mathrm{Fe}$, are negligible for $\mathrm{Ni}$.

In this work we carried out an extensive study of interstitial cluster/dislocation loop mobility in Fe-Ni alloys using different atomic level techniques to discover the strong effect of $\mathrm{Ni}$ on SIA cluster trapping and reducing their mobility and used the results to predict qualitatively some properties of irradiated Fe-Ni ferritic alloys. The structure of the paper is the following. In Section 2 we describe briefly the atomistic techniques used. The results describing $\mathrm{Ni}$ effects on interstitial clusters mobility are presented in Section 3 and the predicted effects under irradiation are summarized and discussed in Section 4.

\section{Modeling approaches.}

We have used molecular dynamics (MD) and molecular statics (MS) techniques. Conventional MD was used to model thermally activated motion, e.g. diffusion of interstitial clusters in bcc-Fe with different $\mathrm{Ni}$ content, from 0.8 to $2.5 \mathrm{at} . \%$ over a wide temperature range from 300 to $900 \mathrm{~K}$. A detailed description of our diffusion modeling approach for clusters performing 1-D motion can be found in previous work on pure Fe [20, 21] and Fe-Cr alloys [22].

The system size for the MD study varied from $\sim 15000$ to $\sim 45000$ mobile atoms depending on 
the cluster size (NSIA) that varied in the range of $7-61$ SIA. We have modeled diffusion up to a few hundred nanoseconds to accumulate statistically significant number of cluster jumps and long enough trajectories. The MD results were used to determine the diffusion coefficients by different methods as described in [23] and applied in [20, 21]. According to [23], the cluster diffusion coefficients can be obtained by treating cluster jumps and their correlations or decomposing their trajectories according to jumps (trajectory jump decomposition -TJD) or time segments (trajectory time decomposition - TTD). If the simulation of each trajectory is long enough, all diffusion coefficients are similar as demonstrated in [20-23]. Ni atoms introduce new correlations in the motion of SIA clusters. This makes treatment of one-dimensional cluster motion more complicated for the segments in the trajectory decomposition should be longer to include these correlations. For example, TTD treatment for 19SIA cluster at 500K converges with segments above $\sim 30$ ps [21]. Treatment of the same cluster trajectory in $\mathrm{Fe}-0.8$ at.\% $\mathrm{Ni}$ alloys demands segments of $\sim 280-300$ ps that is ten times longer. Therefore to achieve the same accuracy of the cluster diffusion coefficient in an alloy one needs to increase the modeling time by the corresponding factor. The effective migration energy, $E_{m}^{*}$, can then be estimated from the Arrhenius-type plot. We also have analyzed particular mechanisms using visualization methods.

MS simulations were carried out in systems from $\sim 230000$ to $\sim 627000$ atoms. MS was used to calculate the cluster-Ni interaction energy by relaxing particular configurations. However, particular impurity distributions can be quite different and lead to quite different total interaction energies and migration barriers especially in concentrated alloys. We therefore used two MS-based approaches.

In the one, the elementary contributions to the total energy state of the cluster in the alloy were calculated by locating a $\mathrm{Ni}$ atom in a position in the atomic rows normal to the cluster habit 
plane. Cluster-Ni binding energy versus row position and distance to the cluster habit plane were obtained.

In the other, we sampled different configurations over a particular Fe-Ni alloy using the approach developed in [22]. In this model, a crystal, with randomly distributed Ni atoms with a particular concentration, $C_{N i}$, was simulated. The SIA cluster was then inserted in the crystal and relaxed. Then the cluster position was shifted by the Burgers vector, $\boldsymbol{b}=1 / 2<111\rangle$, and the system was relaxed again. The process continued until all the configurations along the $\langle 111\rangle$ directions were tested. If the sampled path is long enough all the possible configurations are tested. Here we studied $N=1000$ positions (crystal length along <111> direction was $1000|\boldsymbol{b}| \approx 250 \mathrm{~nm}$ ). The effective binding energy, $E_{N S I A}^{e f f}\left(C_{N i}\right)$, depends on the temperature $T$ and is defined as:

$$
E_{N S I A}^{e f f}\left(C_{N i}\right)=k T \ln \left[\frac{\sum_{i}^{N} \exp \left(E_{i}-E_{\min } / k T\right)}{N}\right]
$$

where $k$ is the Boltzmann constant, $E_{\min }$ and $E_{i}$ are the minimum and the current potential energies calculated after each relaxation in the system containing a NSIA cluster in the alloy with $C_{N i} \mathrm{Ni}$ concentration. In this approach the true migration energy also includes the local barrier for the cluster migration so that the effective migration energy is:

$$
E_{N S I A}^{m}\left(C_{N i}\right)=E_{N S I A}^{e f f}\left(C_{N i}\right)+E_{N S I A}^{m}\left(C_{N i}\right)
$$

Here we did not obtain $E_{N S I A}^{m}$ for each configuration instead we used the corresponding barriers in pure Fe, i.e. for $C_{N i}=0$, from [22] understanding that this is the lower value approximation.

Such a combination of techniques is necessary when a wide range of $\mathrm{Ni}$ concentrations is studied. MD provides robust results for small cluster dynamics in dilute alloys when the total interaction is weak and migration energy is not too high. However, at high concentration the migration energy for large clusters can increase well above $1 \mathrm{eV}$ which makes $\mathrm{MD}$ ineffective in 
accumulating statistics of cluster jumps. Sampling multiple configurations provides statistically

averaged values of $E_{N S I A}^{e f f}\left(C_{N i}\right)$ suitable for estimations of diffusion coefficients. It is important to provide a cross-verification of techniques that is possible for small clusters in dilute alloys.

\section{RESULTS}

\subsection{Dynamics of interstitial clusters in Fe-Ni alloys.}

MD has been successfully applied for modeling small clusters, NSIA $\leq 19$, in low-Ni alloys, $\mathrm{C}_{\mathrm{Ni}} \leq 2.5$ at.\%. For these cases modeling over a few hundred nanoseconds provides robust and statistically significant results for estimating diffusion coefficients, i.e. error bars for these cases are visually smaller than symbols on plots. The results for 7 and 19SIA in $\mathrm{C}_{\mathrm{Ni}}=0.8$ and 2.5 at.\% alloys are presented in Fig.1. One can note the linear plots for pure Fe and 0.8 at.\% Ni alloy and strongly curved behavior for 2.5 at. $\% \mathrm{Ni}$ alloy. Both the diffusion coefficients and migration energies, estimated by fitting to the Arrhenius law and indicated in the Fig.1, demonstrate a visible effect of increasing $\mathrm{Ni}$ content. Thus, at low temperature the diffusivity drops $\sim 100$ times for the 7SIA and 19SIA cluster while their migration energy increases from 0.05 and $0.04 \mathrm{eV}$ in pure $\mathrm{Fe}$ to 0.18 and $0.25 \mathrm{eV}$ in 2.5 at. $\% \mathrm{Ni}$ alloy. Larger clusters were simulated only in the lowest Ni content alloy. Even in this case a very long simulation time is necessary to obtain a reasonably accurate event statistics and this, together with the increased system size for large clusters, demanded very large computational resources. The results for 0.8 at. $\% \mathrm{Ni}$ alloy are presented in Fig.2. At the highest temperature, 900K, all diffusivities differ by $\sim 3-4$ times while at the lowest, $300 \mathrm{~K}$, the difference is above two orders of magnitude. This is mainly due to a significant decrease in low temperature mobility. In fact we could not observe a long-range motion of $\geq 37$ SIA clusters in $\geq 2.5$ at. $\% \mathrm{Ni}$ alloys at medium and low temperature even up to a 
few hundred ns. A visualization analysis shows that clusters of 37 and 61 SIAs move forward and backward over a limited distance between particular configurations in a kind of oscillating manner. Decomposition of their trajectory formally results in a certain value of the diffusion coefficient however no long range motion of these clusters was observed at $\mathrm{T} \leq 600 \mathrm{~K}$. This means that their migration involves much longer correlations, of the order of many tens nanoseconds, and its treatment within conventional MD demands two-three orders of magnitude longer simulation time compared to that applied in this work. Such a pinning is due to the high binding energy of particular configurations, as addressed below. At high temperature these clusters can unpin and move in a smoother way as reflected in Fig.2.

\subsection{Interaction between interstitial clusters and $\mathrm{Ni}$ atoms.}

In this section we present MS results on the interaction between SIA clusters and Ni atoms. Fig.3 shows schematically a hexagonal cluster of 61 SIAs. The numbered positions indicate the intersection of atomic rows perpendicular to the cluster habit plane in the $\langle 111\rangle$ direction along which we calculated interaction energy as a function of the distance from the substituted Ni atom to the cluster habit plane. The results for 7 and 61 SIA clusters are presented in Fig.4. These data show that Ni-substitution has a positive binding energy inside the cluster glide prism, i.e., within the compression region. There is no possibility to verify these properties directly with either experiment or first principle modeling. However, recent progress in density functional theory (DFT) based on the locally self-consistent multiple scattering method (LSMS) makes it possible for the $1 / 2<111>(110)$ edge dislocation [19]. A combination of DFT+LSMS and MS was used in [19] to model interactions between some solutes, including $\mathrm{Ni}$, with edge and screw dislocations. In the case of $\mathrm{Ni}$ the maximum binding was found to be $0.19 \mathrm{eV}$ in the site with the maximum compressive pressure. The same site also had the maximum binding energy, $0.22 \mathrm{eV}$, when an 
infinite edge dislocation was modeled with the current set of interatomic potentials. These energies are very close and we therefore accepted the above results for $\mathrm{Ni}$ - SIA cluster interaction as the most accurate possible to date. This interaction increases the cluster effective migration energy, $E_{m}^{*}$, when it glides through the Fe-Ni alloy matrix. In the first approximation, $E_{m}^{*}=E_{m}+E_{b}$, where $E_{m}$ is the cluster migration energy in pure Fe and $E_{b}$ is the local binding energy. This can be applied directly for the case of dilute alloys when only one Ni atom interacts with the small cluster. In principle, with all interaction energies similar to those presented in Fig.4, it is possible to estimate the total effect that includes the statistics of Ni distribution for large clusters and concentrated alloys. However, because of the great variety of local configurations and possible fluctuations in Ni concentrations such a model would be either quite complicated or too approximate. We therefore applied the MS-sampling technique described in Section 2 to account for the statistically averaged interactions of some clusters and alloys. The results for $N S I A=7,19$ and 37 are presented in Fig.5 showing $E_{m}^{*}$ as a function of $\mathrm{Ni}$ concentration. The results for 7 and 19 SIA in the 0.8 and 2.5 at.\% Ni alloys are very close to the MD data (solid circles in Fig.5), thus validating the main approximations of the model. Fig.5 therefore gives a quite accurate estimation of the statistically averaged effective migration energy for clusters in alloys which are not amenable to the conventional MD modeling. Using this one can estimate the cluster effective diffusion coefficient $D^{*}{ }_{\text {NSIA }}$ of NSIA interstitials in the random alloys with $C_{N i}$ concentration:

$$
D_{N S I A}^{*}\left(C_{N i}\right)=D_{N S I A}^{* 0} \exp \left(\frac{E_{N S I A}^{*}\left(C_{N i}\right)}{k T}\right) .
$$

The main issue here is the pre-exponential factor $D_{N S I A}^{* 0}$ which, in general, depends on NSIA and $C_{N i}$. It also may depend on other factors such as temperature (see Fig.1 where the cluster 
diffusivity deviates from the Arrhenius law). We introduce the reduced mobility factor, $B_{N S I}$, defined as the ratio of the cluster diffusion coefficient in an alloy over that in pure Fe:

$$
B_{N S A}\left(C_{N i}\right)=D_{N S I A}^{*}\left(C_{N i}\right) / D_{N S I A}^{*}\left(C_{N i}=0\right) .
$$

Assuming that some contributions to the cluster pre-exponential factors may have similar dependences in pure $\mathrm{Fe}$ and $\mathrm{Fe}-\mathrm{Ni}$ alloy, the errors cancel and this factor is more accurate than the diffusion coefficients themselves.

The results for $N S I A=7,19$ and 37 with $C_{N i}=2$ at $\%$ and 10at.\% are presented in Fig.6. For cross-verification issues, we also present MD results for 7 and 19 SIA clusters in Fig.6a which are very close to MS-sampling. The results presented in Fig.6 for large clusters in concentrated alloys provide information that would not be possible to obtain in direct MD modeling of thermally-activated SIA cluster motion through the Fe-Ni alloys. The 37SIA cluster in the 2.5at.\% Ni alloy had its mobility reduced by seven orders in magnitude at room temperature and more than 1000 times at $600 \mathrm{~K}$ (Fig.6a). All effects are much stronger at 10at.\% Ni as seen in Fig.6b. We did not study $C_{N i}>10$ at.\% for we wanted to consider only ferritic alloys.

\section{Discussion.}

The main results obtained in the present research are summarized in Fig.6 demonstrating the effect of Ni impurities in reducing SIA cluster mobility in Fe-Ni alloys in some particular cases. The issues of modeling and statistical treatment were discussed above and here we discuss only the implications of the results received.

The reduction of the SIA cluster diffusivity in a Fe-Ni solid solution is related eventually to its interaction with $\mathrm{Ni}$ atoms. Ni exhibits an attractive interaction with the compressed region of the dislocation loop, which practically vanishes to zero in the loop center. In a random solid 
solution, the interaction energy depends strongly on the number of $\mathrm{Ni}$ atoms 'in contact' with the loop core inside the glide prism, which increases with NSIA and $C_{N i}$. However, the increase of the apparent migration barrier, i.e. the value extracted from the MD simulations, is defined not by the absolute value of the interaction energy $E_{N S I A}^{e f f}\left(C_{N i}\right)$ but by its variation over the alloy volume (see Eq.(2)). The stronger the variance, the higher the effective migration barrier $E_{N S I A}^{m}\left(C_{N i}\right)$ (see Eq.(3) and [22] for Fe-Cr solid solution). Apparently, the value of $E_{N S I A}^{m}\left(C_{N i}\right)$ is high enough to observe the total suppression of 37SIA cluster mobility in MD simulations at $\mathrm{T} \leq 600 \mathrm{~K}$ in $\mathrm{Fe}-0.8 \% \mathrm{Ni}$ alloy. Note that these results should be taken as rather accurate due to the positive verification by DFT modeling for the edge dislocation described above.

For a better understanding of the role of $1 \mathrm{D}$ glissile SIA clusters in radiation damage of Fe-Ni alloys, we first summarize relevant information obtained in previous extensive modeling of high-energy displacement cascades [24-28] and defect cluster properties [21, 25,30-32] in Fe:

- Self-primary knock-on-atoms, which reproduce the neutron irradiation case, with energy above $\sim 5 \mathrm{keV}$ produce displacement cascades. The maximum energy cascades are about $20-25 \mathrm{keV}$ whereas at higher energy extensive formation of sub-cascades is observed.

- $25 \mathrm{keV}$ cascades produce on average $\sim 75$ Frenkel pairs (FPs) although some cascades produce more than $100 \mathrm{FPs}$. Clusters of up to a few tens SIAs form directly in cascades [24-28].

- Clusters of $N S I A \geq 7$ are in fact $1 / 2<111>$ dislocation loops that perform fast 1 -D thermally activated glide with a very low activation energy $E_{m}^{*}<0.1 \mathrm{eV}$.

- The fraction of SIAs produced in the form of glissile clusters is $\varepsilon_{i}^{g} \approx 0.1-0.35$ depending on the ambient temperature, interatomic potentials used, PKA energy, etc. Further cascade annealing results in additional recombination reducing this fraction closer to the experimentbased estimations, $\sim 0.1[33]$. 
These findings in conjunction with the modern theory for radiation damage evolution [3], enabled some long-standing experimental observations to be understood and rationalized selfconsistently. One of the most important theoretical results is understanding the effect of recoil energy difference between electron, ion and neutron irradiation [4] where the main role is attributed to the formation of 1-D glissile SIA clusters in high energy cascades. The direct connection between these features and swelling under neutron irradiation is expressed in Eq.1 above. However, the consequences of the clusters are not limited only by swelling. 1-D gliding clusters reduce the number of dislocation loops (so-called matrix damage) for they are primary absorbed on grain boundaries and dislocations. These clusters also decorate pre-existing dislocations thus immobilizing them and, according to the cascade induced source hardening (CISH) model $[32,34]$ increasing the yield stress in irradiated materials. Also, $1 / 2<111>$ glissile loops interacting with each-other create $<100>$ loops by the mechanism described recently [35]. Such loops are frequently observed in Fe and some ferritic alloys (see discussions in [35, 36]).

The results presented in Fig. 6 demonstrate that by adding Ni impurity to Fe the mobility of SIA clusters can be reduced up to complete immobilization depending on cluster size, $\mathrm{Ni}$ content and ambient temperature. Under irradiation this can lead to the following effects:

First, due to their reduced mobility, the SIA clusters will participate in recombination reactions with vacancies thus reducing the production bias (Eq.1) and therefore the total swelling of the material. This effect can be very strong because, as demonstrated earlier, the interaction of vacancies with small SIA clusters is much stronger than that with dislocations [37]. This is due to the high pressure inside the cluster glide prism and high cluster mobility.

Second, reduction in the vacancy supersaturation level and corresponding decrease in swelling, reduces the contribution of voids to hardening. However, $1 / 2<111>$ clusters left in the 
bulk and possibly decorated by $\mathrm{Ni}$ atoms may contribute significantly in matrix hardening and compensate for the loss of the void contribution.

Third, concentration of $1 / 2<111>$ dislocation loops should be increased in comparison with pure Fe where a vast majority of them are absorbed at grain boundaries and dislocations by 1-D glide. Consequently the mean cluster size should be smaller than in pure Fe where small clusters glide out and only large clusters formed by cluster-cluster coalescence are left due to their reduced mobility.

Fourth, according to [35] reactions between $1 / 2<111>$ clusters/loops leading to the formation of $\langle 100\rangle$ clusters occur in a rather narrow size range, NSIA 30-50. Therefore immobilization of these clusters reduces the probability of $<100>$ cluster/loop formation

Fifth, slowing down or immobilizing the glissile clusters should reduce or even prevent dislocation decoration and thus reducing the $\mathrm{CISH}$ contribution $[32,34]$ which is a positive factor in view of the plastic instability observed in irradiated materials beyond a certain dose.

So far, not much is known experimentally about the role of $\mathrm{Ni}$ in radiation induced microstructure evolution. Neutron irradiated pure $\mathrm{Fe}$ and $\mathrm{Fe}-0.7 \mathrm{Ni}$ were studied in [13]. The damage microstructure included $\langle 100\rangle$ and $1 / 2<111>$ interstitial dislocation loops, whose size, number density and relative fraction were found to be affected by Ni. For example, in pure Fe $90 \%$ of the loops were of $<100>$ type whereas in the Fe-Ni alloy $75 \%$ of loops had $\boldsymbol{b}=1 / 2<111>$ but their number density increased and size decreased. More recently, pure Fe and a number of RPV-model alloys (including Fe with 1, 2, 3 and 5 wt.\% Ni and Fe-0.2C-1Ni) were irradiated by neutrons and their microstructure was studied [38]. In pure Fe, the vast majority of SIA dislocation loops were of $\langle 100\rangle$ type with only a few loops with $\boldsymbol{b}=1 / 2<111>$. Voids were also observed in agreement with previous experimental studies [39]. However, unlike in pure Fe, a 
dense population of fine, hardly resolvable features was observed in the Fe-Ni alloys. These features are expected to be $1 / 2<111>$ loops. Voids were not observed by TEM in the Fe-Ni alloys. TEM studies also concluded a general decrease of the radiation damage in higher Ni content alloys. Consistently with the TEM observations radiation induced increase of tensile strength and micro-hardness was found to be weaker in higher-Ni alloys.

The main observations in the above mentioned experiments are fully consistent with the implications resulting from the immobilization of $1 / 2<111>$ loops by Ni atoms. However, in-depth understanding of how $\mathrm{Ni}$ affects radiation damage in ferritic alloys needs more experimental work. The list of effects and predictions presented above is incomplete and can be expanded even towards quantitative predictions if the approach suggested here is extended to more complicated systems and combined with the modern theory of radiation damage in materials.

\section{Conclusions}

The set of atomistic techniques and the accuracy of the interatomic potentials used in this research allowed reliable results to be obtained on the influence of $\mathrm{Ni}$ solute atoms on the interstitial cluster/dislocation loop mobility in Fe. These results, in turn, can fit properly in the modern approach to the generalized radiation damage theory that should be able to complement

realistically the lack of experimental data. The predictions derived here may be useful in designing future experiments.

The main points of the paper can be summarized as following.

1. The case of Fe-Ni alloys was chosen because of: i) availability of accurate interatomic potentials for $\mathrm{Fe}-\mathrm{Ni}$ system; ii) the need for a highly predictive theory due to a 
significant lack of experimental data and iii) expectations found in the literature that these alloys have potential for future nuclear energy applications.

2. Three atomic-scale modeling techniques were used to study mobility of clusters of 7 to 61 interstitials in Fe alloys with $\mathrm{Ni}$ concentrations from 0.8 to 10 at.\%.

3. Molecular dynamics was used to model thermally activated motion directly in the case of small clusters and low $\mathrm{Ni}$ concentration. Direct interactions between Ni-atoms and interstitial clusters were studied in the Fe matrix by molecular statics. MS-based sampling and statistical treatment of multiple configurations in alloys were used to account for the statistics of interstitial cluster configurations in particular Fe-Ni alloys.

4. This combination of techniques, being cross-verified, allowed data to be obtained for macroscopic cluster behavior with high accuracy at atomistic level.

5. Effective activation energies for cluster motion obtained for different clusters and alloys indicate that $\mathrm{Ni}$ atoms suppress cluster mobility up to complete immobilization. The effect is stronger for larger clusters and higher Ni content.

6. The results obtained provide a scenario explaining the differences in the microstructural evolution between pure bcc $\mathrm{Fe}$ and $\mathrm{Fe}-\mathrm{Ni}$ alloys observed in the scarce experimental studies and may help in designing the new experiments.

\section{Acknowledgements}

The authors wish to acknowledge Drs. R. Stoller, A. Barashev and L. Malerba for the useful discussions.

Research at the Oak Ridge National Laboratory supported as part of the Center for Defect Physics, an Energy Frontier Research Center funded by the U.S. Department of Energy, Office 
of Science, Office of Basic Energy Sciences. Research at UPC was supported by EC (FP7, PERFORM60: 232612) and MINECO (FIS2012-39443-C02-02). Research at SCK·CEN was partially supported by EUROfusion consortium. This work also contributes to the Joint Program on Nuclear Materials (JPNM) of the European Energy Research Alliance (EERA).

*) Corresponding author: Materials Science and Technology Division, Oak Ridge National Laboratory, Oak Ridge, TN 37831, USA.

Phone: (1) 8655763254

e-mail: osetskiyyn@ornl.gov. 


\section{References}

1. Odette GR, Lucas GE. JOM, 2001;53:18.

2. Dudarev SL, Boutard JL, Lässer R, Caturla MJ, Derlet PM, Fivel M, Fu CC, Lavrentiev MY, Malerba L, Mrovec M, Nguyen-Manh D, Nordlund K, Perlado M, Schäublin R, Van

Swygenhoven H, Terentyev D, Wallenius J, Weygand D, Willaime F. J Nucl Mat 2009; 386388: 1 .

3. Golubov SI, Barashev AV, Stoller RE, Radiation Damage Theory. In: Konings RJM, (ed.) Comprehensive Nuclear Materials, volume 1, pp. 357-391, Amsterdam, 2012: Elsevier. http://info.ornl.gov/sites/publications/files/Pub28175.pdf.

4. Singh BN. Nature $1973 ; 224: 142$.

5. Hudson, JA, Mazey, DJ, Nelson RS. J Nucl Mater, 1971;41:241.

6. Norris DIR. J Nucl Mater 1971; 40:66.

7. Farrell K, Houston JT, Wolfenden A, King RT, Jostons A. In Radiation-Induced Voids in Metals, CONF-710601; Corbett JW, Ianniello LC, Eds.; National Technical Information Service: Springfield, VA, 1972; pp 376-385.

8. Zinkle SJ, Farrell K. J Nucl Mater 1989;168:262.

9. Golubov SI, Barashev AV, Stoller RE. On the Origin of Radiation Growth of HCP Crystals, ORNL/TM-2011/473.

10. Golubov SI, Barashev AV, Stoller RE, Singh BN. "Breakthrough in Understanding Radiation Growth of Zirconium, in: Proceeding of 17th International Symposium "Zirconium in the Nuclear Industry", ASTM STP 1543, R.J. Comstock, Ed., ASTM International, 2014, in press.

11. Norgett MJ, Robinson MT,Torrens IM. Nucl Eng Design 1975:33,50; Standard E521, 
ASTM Annual Book of Standards, 1989.

12. Odette GR. Scripta Metallurgica 1983;17:1183.

13. Hoelzer DT, Ebrahimi F. Mat Res Soc Symp Proc, 1995;373:57.

14. Kočík J, Keilová E, Brumovský M. PERFECT_Report:D1-3.8.

15. ASTM Standard E 900, 20022007 Predicting Radiation-Induced Transition Temperature Shift in Reactor Vessel Materials, E706 (IIF) (West Conshohocken, PA: ASTM International).

16. Mendelev M, Han A, Srolovitz D, Ackland G, Sun D, Asta M. Phil Mag 2003; A83:3977

17. Bonny G, Pasianot RC, Malerba L. Modelling Simul Mater Sci Eng 2009;17,025010

18. Voter AF, Chen SP. Mater Res Soc Symp Proc 1987;82:175

19. Samolyuk G, Odbadrakh., Kh, Osetsky YN, Stoller R, Nicholson D and Stocks G M," Interaction of $\mathrm{Cr}$ and $\mathrm{Ni}$ solute atoms with core of $1 / 2<111>$ screw and edge dislocations in bcc Fe”, Center for Defect Physics, Oak Ridge National Laboratory, unpublished. Pseudopotentials + plane wave DFT approach was used to verify LSMS in modeling Ni and Cr interacting with defects in Fe in small, $100-200$ atoms, systems. LSMS + MS then was used to model interaction with edge and screw dislocations in $\sim 1200$ atoms system.

20. Terentyev D, Malerba L, Hou M. Phys Rev B 2007;75:104108.

21. Anento N, Serra A, Osetsky YN. Modelling Simul Mat Sci Eng 2010;18:025008.

22. Terentev D, Malerba L, Barashev AV. Philos Mag 2008:88:21.

23. Osetsky YN. Defects and Diffusion in Metals - Annual Retrospective III, 2001, Defect and Diffusion Forum, 2001, 188-190, 71.

24. Bacon DJ, Osetsky YN, Stoller RE, Voskoboinikov RE. J Nucl Mater 2003;323:152.

25. Stoller RE, Odette GR, Wirth BD, J Nucl Mater 1997; 251: 49. 
26. Stoller RE. "Primary Radiation Damage Formation," in Comprehensive Nuclear Materials, Konings RJM, Allen TR, Stoller RE, Yamanaka S. Eds., Elsevier Ltd., Amsterdam, 2012, pp. 293-332.

27. Souidi A, Becquart CS, Domain C, Terentyev D, Malerba L, Calder AF, Bacon DJ, Stoller RE, Osetsky YN, Hou M. J Nucl Mater 2006:355:89.

28. Calder AF, Bacon DJ, Barashev AV, Osetsky Yu N. Phil Mag Lett 2008:88:43.

29. Osetsky YN, Serra A, Singh BN, Golubov SI. Phil Mag A 2000:80:2131.

30. Barashev AV, Osetsky YN, Bacon DJ. Phil Mag A 2000:80:2709.

31. Osetsky YN, Bacon DJ, Serra A, Singh BN, Golubov SI. Phil Mag A 2003;83: 61.

32. Singh BN, Foreman AJE, Trinkaus H. J Nucl Mater 1997;249:103.

33. Zinkle SJ, Singh BN. J Nucl Mater 1993;199:173.

34. Trinkaus H, Singh BN, Foreman AJE. J Nucl Mater 1997;249: 91.

35. Xu H, Stoller RE, Osetsky YN, Terentyev D. Phys Rev Lett 2013;110:265503.

36. Terentyev D, Bergner F, Osetsky YN. Acta Mat 2013;61:1444.

37. Pelfort M, Osetsky YN, Serra A. Phil Mag Lett 2001;81:803; Puigvi MA, Osetsky YN. and Serra A, Materials Sci. and Eng., 2004; A365: 101.

38. Achievements within the FP6/PERFECT project, J Nucl Mater 2010;406:1-204.

39. Hernández-Mayoral M, Gómez-Briceño D. J Nucl Mater 2010;399:146. 


\section{Figure captions}

Fig.1. Diffusion coefficients of 7 and 19 SIA clusters in pure Fe and alloys with 0.8 and 2.5 at.\% Ni calculated by time trajectory decomposition (TTD) method. The average migration energies are indicated on the top.

Fig.2. Diffusion coefficients of SIA clusters estimated by MD in Fe-0.8at.\% Ni alloy compared with those in pure Fe.

Fig. 3. Schematic presentation of a 61-site hexagon in $\{111\}$ plane. SIA cluster was created by splitting the shown lattice sites along the $\langle 111\rangle$ directions perpendicular to the plane. After relaxation this results in a prismatic dislocation loop with Burgers vector $1 / 2<111>$ and $\{111\}$ habit plane. Numbered sites indicate cross-sections with atomic rows where Ni substitution was inserted to define cluster-Ni binding energy.

Fig.4. Dependence of Ni-substitution binding energy on the distance to the 7 and 61 SIA cluster habit plane along <111> atomic rows indicated in Fig.3. Circle symbols indicate sites inside the cluster glide prism whereas triangular symbols those outside.

Fig.5. Effective cluster migration energy estimated by MD (full symbols) and by MSsampling (open symbols) as function of Ni concentration in $\mathrm{Fe}-\mathrm{Ni}$ random alloys

Fig.6. Temperature dependence of the reduced mobility factor estimated for different clusters in Fe-Ni alloys with a) 2 at.\% Ni and b) 10 at.\% Ni. Fig.6a also contains MD obtained values for $N S I A=7$ and 19 (solid symbols). 
The Role of Nickel in Radiation Damage of Ferritic Alloys.

Y.Osetsky, N.Anento, A.Serra and D.Terentyev

FIGURES

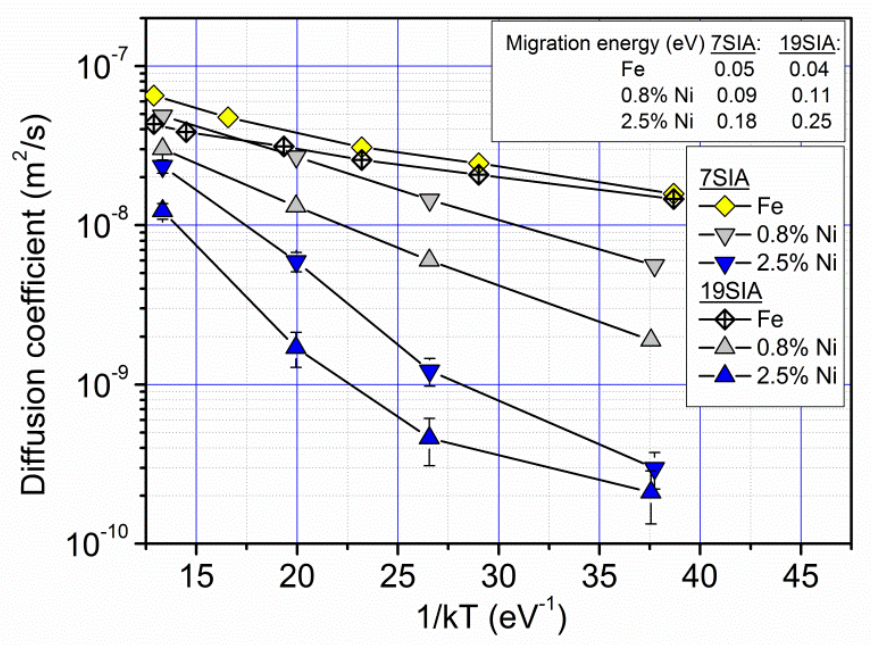

Figure 1

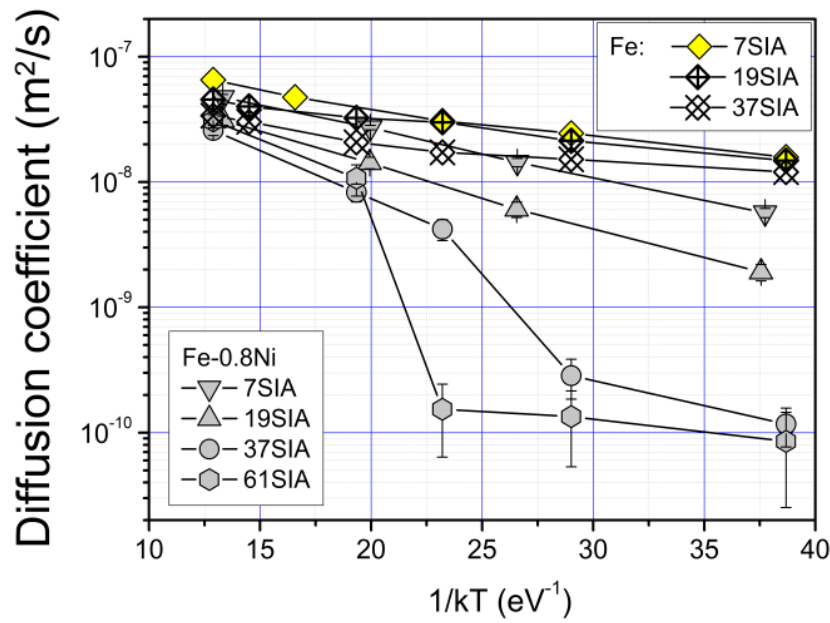

Figure 2. 


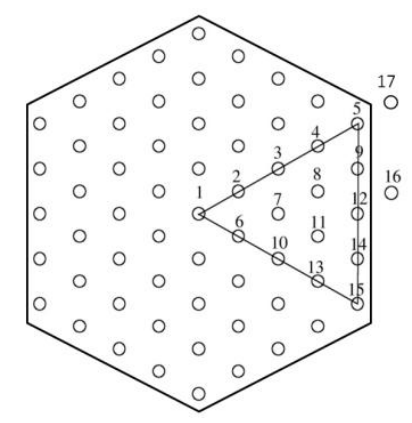

Figure 3

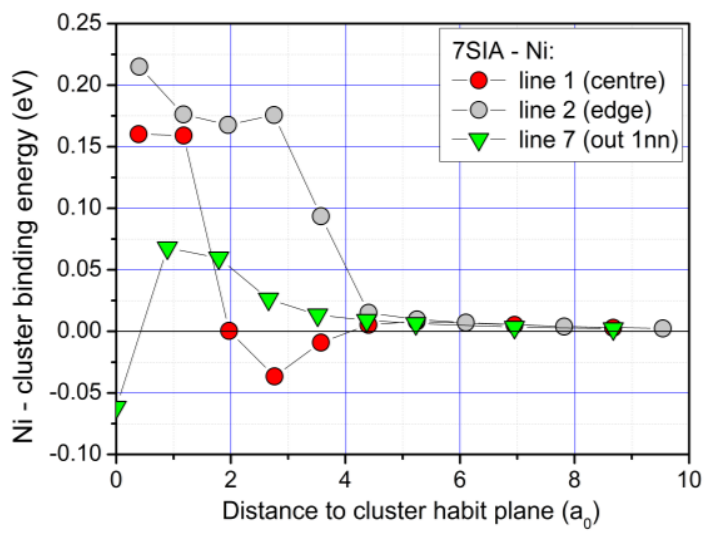

a)

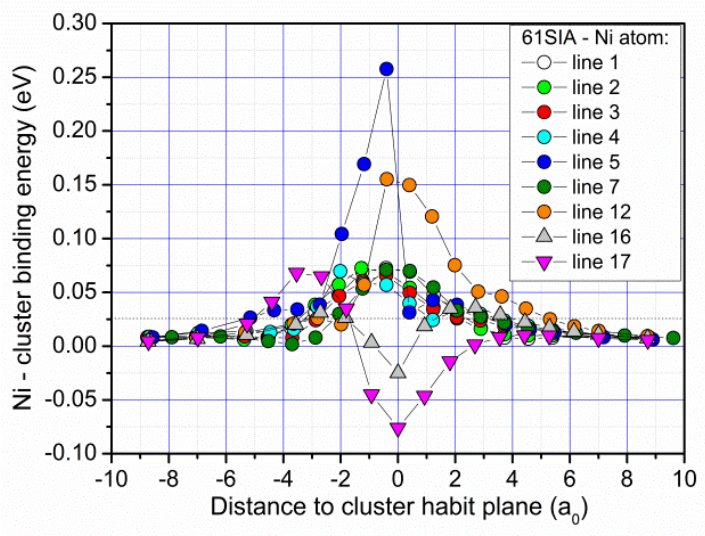

b)

Figure 4 


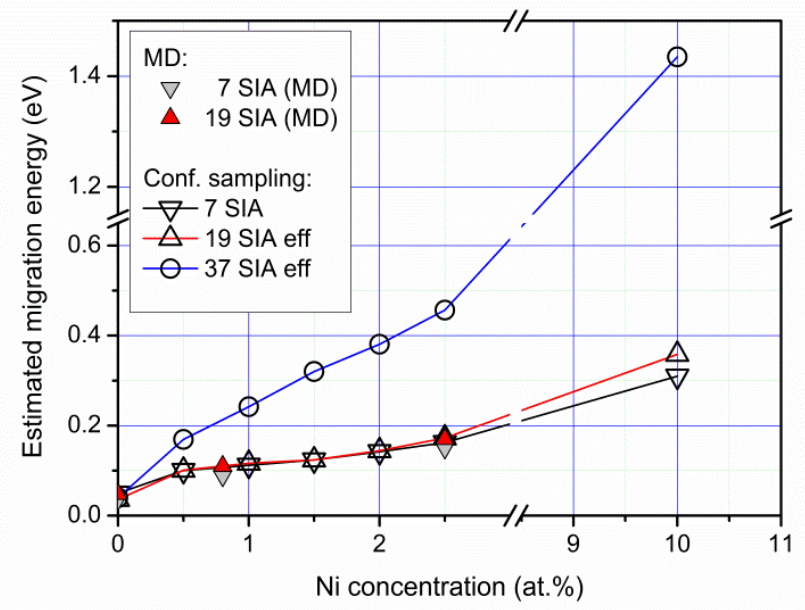

Figure 5

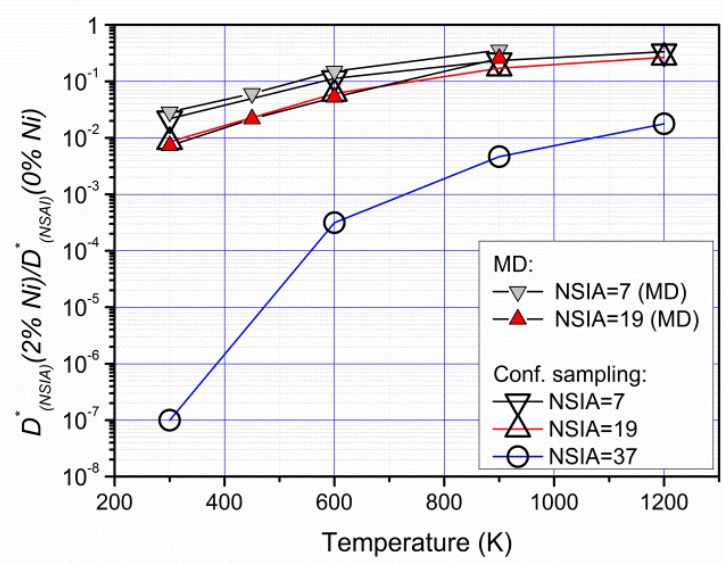

(a)

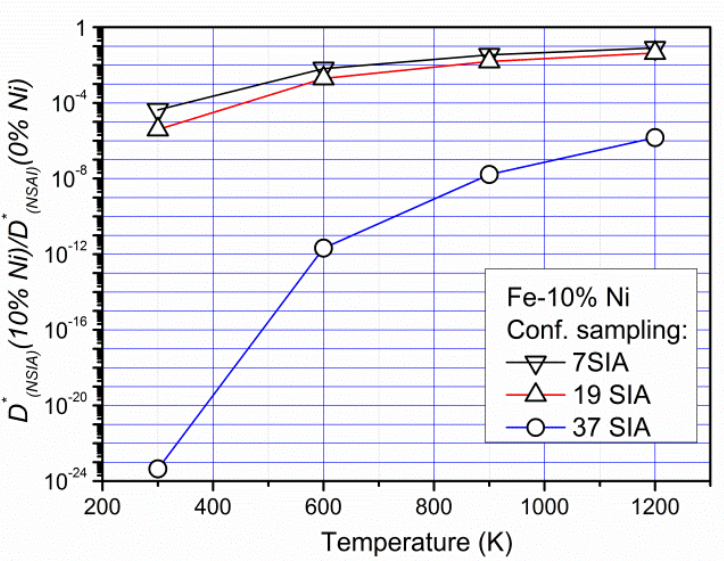

(b)

Figure 6. 